\title{
Hipovitaminosis D en niños y adultos en un prestador de salud del Uruguay y su relación con el uso de antiepilépticos
}

The effect of antiepileptic drugs in adult and child hypovitaminosis D in a Uruguayan health institution

Hipovitaminose $D$ em crianças e adultos em um provedor de saúde uruguaio e sua relação com o uso de medicamentos antiepilépticos

Valentina Catenaccio¹, Silvia Xavier², Agustina De Santis ${ }^{3}$, Noelia Speranza", Andrés Bálsamo ${ }^{5}$, Florencia Galarraga ${ }^{6}$, María Eugenia Chaibun7, Luciana Salazar ${ }^{7}$, Laura Sola ${ }^{9}$, Stella Gutiérrez ${ }^{10}$, Ricardo Hermo ${ }^{11}$, Gustavo Tamosiunas ${ }^{12}$

\section{Resumen}

Introducción: la hipovitaminosis D se encuentra ampliamente extendida a nivel mundial, con consecuencias clínicas a nivel óseo y extraóseo. Entre los factores que la causan se encuentran los antiepilépticos (AE). En Uruguay no se conoce su prevalencia en niños ni en pacientes que reciben $A E$.

Objetivos: conocer la prevalencia de hipovitaminosis D de niños y adultos en un prestador de salud y compararla con la prevalencia en pacientes bajo tratamiento con $\mathrm{AE}$.

Método: estudio descriptivo, transversal, realizado entre marzo y diciembre de 2017. Las variables analizadas fueron: niveles de vitamina D, calcio, fósforo, fosfatasa alcalina y parathormona intacta. Se consideró insuficiencia de vitamina D niveles menores de $30 \mathrm{ng} / \mathrm{ml}$ y déficit niveles menores de $20 \mathrm{ng} / \mathrm{ml}$.

Resultados: se incluyeron 113 pacientes, 60 niños y 53 adultos. La prevalencia global de insuficiencia de vitamina D fue de 89\% y déficit de 60\%. En niños expuestos a AE, la media de vitamina D fue 17,5 ng/ml, y en niños no expuestos 19,6 ng/ml. En adultos la media de vitamina $D$ fue de 18,1 en expuestos a AE y 16,9 en no expuestos. La diferencia de medias no fue estadísticamente significativa en niños ni en adultos. Se observaron niveles de calcemia significativamente descendidos en niños y adultos con $A E$.

\footnotetext{
1. Profesora adjunta del Departamento de Farmacología y Terapéutica, Facultad de Medicina, Universidad de la República.

2. Ex Asistente del Departamento de Farmacología y Terapéutica, Facultad de Medicina, Universidad de la República.

3. Asistente del Departamento de Farmacología y Terapéutica, Facultad de Medicina, Universidad de la República.

4. Profesora agregada del Departamento de Farmacología y Terapéutica, Facultad de Medicina, Universidad de la República.

5. Profesor adjunto Medicina Preventiva y Social, Facultad de Medicina, Universidad de la República.

6. Asistente del Departamento de Farmacología y Terapéutica, Facultad de Medicina, Universidad de la República.

7. Asistente del Departamento de Neuropediatría, Facultad de Medicina, Universidad de la República.

8. Asistente del Departamento de Neuropediatría, Facultad de Medicina, Universidad de la República.

9. Profesora adjunta Medicina Preventiva y Social, Facultad de Medicina, Universidad de la República.

10. Ex Prof. Agda. de Pediatría Facultad de Medicina, Universidad de la República. Jefe del Departamento de Pediatría del CASMU-IAMPP

11. Médico laboratorista del CASMU-IAMPP.

12. Profesor titular del Departamento de Farmacología y Terapéutica, Facultad de Medicina, Universidad de la República.

Departamento de Farmacología y Terapéutica, Facultad de Medicina, Universidad de la República.

El estudio contó con el apoyo financiero de la Comisión Sectorial de Investigación Científica (CSIC) de la Universidad de la República a través del llamado Iniciación a la investigación- modalidad 1, diciembre de 2015.

Los autores declaran no tener conflicto de interés.

Aprobado por el Comité de ética del CASMU.

Correspondencia: Dra. Valentina Catenaccio. Correo electrónico: valentina.catenaccio@gmail.com

Recibido: $17 / 6 / 2020$

Aprobado: 13/1/2021

Attribution-NonCommercial 4.0 International (CC BY-NC 4.0)
} 
Conclusiones: la insuficiencia de vitamina D fue cercana a $90 \%$ y el déficit superó el $50 \%$. No se encontraron diferencias significativas entre grupos en hipovitaminosis $D$, pero se observaron niveles de calcemia reducidos en los expuestos a $A E$. Es necesario continuar analizando los factores que la causan y sus consecuencias clínicas.

\author{
Palabras clave: Hipovitaminosis D \\ Deficiencia de vitamina D \\ Hipocalcemia \\ Anticonvulsivantes \\ Antiepilépticos
}

\section{Introducción}

La vitamina $\mathrm{D}$ es una vitamina liposoluble, considerada también una hormona que regula la homeostasis mineral y mantiene la concentración de calcio dentro de los límites normales. Protege la integridad del esqueleto, modula el crecimiento y la diferenciación celular. Además de su rol en la homeostasis del calcio, la vitamina $D$ regula otras funciones, denominadas funciones extraóseas, con posibles beneficios, como la regulación del sistema inmune, la función muscular, el sistema cardiovascular y metabólico ${ }^{(1,2)}$.

Existe evidencia creciente acerca del déficit de vitamina $\mathrm{D}$ en la población mundial. Según la International Osteoporosis Foundation (IOF) y la National Osteoporosis Foundation (NOF), la hipovitaminosis D se encuentra ampliamente extendida en los seis continentes y está reemergiendo como problema de salud pública glo$\mathrm{bal}^{(1,2)}$. La prevalencia de hipovitaminosis D considerada como menor a $30 \mathrm{ng} / \mathrm{ml}$ en Estados Unidos es de $76 \%$, según datos del Centro Nacional de Salud y Nutrición NANHES (National Health and Nutrition Examination Survey) ${ }^{(3)}$. En España, los estudios de prevalencia realizados en niños escolares entre 9 a 14 años identificaron un déficit de vitamina $D$ entre $45 \%$ y $51 \%$, considerando niveles menores a $20 \mathrm{ng} / \mathrm{ml}^{(4,5)}$. A nivel regional, en Argentina una revisión de 2015 que incluyó 17 estudios realizados en adultos jóvenes, adultos mayores, mujeres premenopáusicas y niños, encontró que el promedio ponderado de prevalencia de déficit fue de $43,3 \%$. Se observó una media de vitamina D entre 6 a 25 $\mathrm{ng} / \mathrm{ml}$, y en la mayoría de los estudios fue menor a 20 $\mathrm{ng} / \mathrm{ml}^{(6)}$. En Uruguay se han estudiado los niveles de vitamina $\mathrm{D}$ en adultos $\mathrm{y}$ en poblaciones especiales como embarazadas, mujeres posmenopáusicas y pacientes en hemodiálisis crónica encontrando niveles de hipovitaminosis menores a $30 \mathrm{ng} / \mathrm{ml}$, cercanos a $90 \%{ }^{(7-11)}$.

Diversos factores pueden influir en los niveles de vitamina $\mathrm{D}$, entre ellos, la baja exposición a la luz solar, la presencia de enfermedades malabsortivas, la obesidad,

$\begin{array}{ll}\text { Key words: } & \text { Hypovitaminosis D } \\ & \text { Vitamin D deficiency } \\ & \text { Hypocalcemia } \\ & \text { Anticonvulsants } \\ & \text { Antiepileptics }\end{array}$

el sedentarismo y también la exposición a fármacos que interfieren en el metabolismo de ésta, por ejemplo los fármacos antiepilépticos $(\mathrm{AE})^{(1,12)}$.

Del punto de vista teórico es conocida la interacción de los AE y el descenso de los niveles séricos de vitamina $\mathrm{D}$, principalmente aquellos inductores de las enzimas hepáticas del citocromo P450 (CYP450), como fenobarbital (FB), difenilhidantoína (DFH), carbamazepina (CBZ) y primidona. Dichos AE estimulan el metabolismo de la vitamina $\mathrm{D}$ con el consecuente descenso de sus niveles plasmáticos. Pero también existe interacción para aquellos $\mathrm{AE}$ no inductores de las enzimas hepáticas, incluso inhibidores de éstas, como el ácido valproico (VPA). Este fármaco produce inhibición de la enzima 25 hidroxilasa, reacción enzimática de bioactivación de la vitamina $\mathrm{D}$. Además de disminuir los niveles de vitamina $\mathrm{D}$, los antiepilépticos alteran otros componentes del metabolismo fosfocálcico, como la disminución de la absorción de calcio, efectos directos sobre las células óseas, deficiencia de calcitonina y resistencia a la hormona paratiroidea ${ }^{(11,13)}$.

Varios estudios observacionales realizados en pacientes en tratamiento con AE han encontrado niveles descendidos de vitamina D y otras alteraciones del metabolismo fosfocálcico, como hipocalcemia o alteraciones de la PTH o fosfato ${ }^{(14-16)}$. Las consecuencias clínicas de estos efectos aún no son claras, si bien existen estudios que han observado osteopenia, osteoporosis y mayor incidencia de fracturas en este grupo de pacientes ${ }^{(12,16)}$. La causalidad, sin embargo, es todavía difícil de establecer, ya que las fracturas pueden deberse a etiologías multifactoriales como, por ejemplo, trauma por convulsión o efectos sedativos de algunos AE con aumento de riesgo de caídas, entre otros.

Además de su rol en la regulación del metabolismo fosfo- cálcico, la vitamina D se considera una neurohormona posiblemente implicada en varias enfermedades extraóseas, como trastornos autoinmunes, cáncer, depresión, diabetes, enfermedades vasculares y neurode- 
generativas, entre otras ${ }^{(17)}$. Si bien aún se encuentra en investigación, se le atribuye a la vitamina $D$ efectos neuroprotectores, con un rol en la neurogénesis y diferenciación neuronal, habiéndose identificado tanto a la vitamina D como a las enzimas CYP450 que la hidroxilan en el sistema nervioso central, con predominio en el hipotálamo. Su posible rol en la epileptogénesis todavía no está aclarado, si bien se encuentran en marcha varios estudios $^{(18)}$.

En Uruguay no existen datos acerca de los niveles séricos de vitamina $D$ en pacientes pediátricos ni tampoco en individuos tratados con $\mathrm{AE}$, ya sea adultos o niños. El objetivo del presente estudio fue conocer la prevalencia de hipovitaminosis $\mathrm{D}$ en niños y adultos en una población de Uruguay asistida en el Centro de Asistencia del Sindicato Médico del Uruguay (CASMU-IAMPP) y compararla con la prevalencia en pacientes de los mismos grupos etarios en tratamiento con AE.

Este estudio se enmarca dentro del convenio del Departamento de Farmacología y Terapéutica de la Facultad de Medicina de la Universidad de la República (Udelar) y la Unidad de Investigación Clínica-CASMU, y contó con el apoyo financiero de la Comisión Sectorial de Investigación Científica (CSIC) de la Udelar a través del llamado Iniciación a la investigación- modalidad 1, diciembre de 2015.

\section{Metodología}

Se realizó un estudio descriptivo, transversal, para estimar la prevalencia de hipovitaminosis $\mathrm{D}$ en niños y adultos asistidos en CASMU-IAMPP, entre el 1/3/2017 al 31/12/2017. Se comparó la prevalencia de hipovitaminosis $\mathrm{D}$ entre individuos que recibían $\mathrm{AE}$ y los que no los recibían. Se incluyeron dos grupos de pacientes. Grupo 1: niños de 2 a 14 años y adultos de 50 a 70 años en tratamiento con $\mathrm{AE}$ asistidos en policlínicas de neuropediatría y neurología de CASMU-IAMPP. Se incluyeron pacientes que tuvieran prescripto un $\mathrm{AE}$, independientemente del motivo de la indicación. Grupo 2: niños de 2 a 14 años y adultos de 50 a 70 años sin tratamiento con $\mathrm{AE}$, entrevistados en sala de espera de laboratorio y carné de salud de CASMU- IAMPP.

Se excluyeron los individuos portadores de insuficiencia hepática severa, insuficiencia renal en diálisis, neurofibromatosis, enfermedades disabsortivas (enfermedad celíaca, colitis ulcerosa, enfermedad de Crohn), pacientes que estuvieran recibiendo vitamina $\mathrm{D}$, corticoides sistémicos por más de tres meses, y medicamentos que interfieran con el metabolismo fosfocálcico, como uso sistémico de ketoconazol, diuréticos tiazidas y rifampicina.

Se solicitó consentimiento para participar del estudio. A los pacientes que aceptaban participar se les reali- zaba un estudio paraclínico adicional (del que ya tenían solicitado por su médico) para dosificar vitamina $\mathrm{D}$, calcio, fósforo, fosfatasa alcalina y parathormona intacta (PTHi) con la muestra de sangre que ya tenía coordinada.

Se aplicó una ficha de recolección de datos preelaborada por los autores para consignar características basales: edad, sexo e índice de masa corporal (IMC) y AE recibidos, duración del tratamiento y valores hallados de metabolismo fosfocálcico.

La edad se consignó en años. El sexo se consideró como femenino o masculino. El IMC se calculó según la razón entre el peso (en kilogramos) y el cuadrado de la talla (en metros) ${ }^{(19)}$.

Los AE se clasificaron según el código del Sistema de Clasificación Anatómica Terapéutica, (ATC) de la Organización Mundial de la Salud(OMS) ${ }^{(20)}$. Se consideró politerapia el uso de dos o más $\mathrm{AE}^{(21)}$.

La duración del tratamiento se dividió en dos categorías: menor de seis meses o mayor de seis meses. Este punto de corte en el tiempo de uso de AE se basó en autores como Borusiak, que consideran como tratamiento prolongado más de seis meses, considerándolo posible indicador de alteraciones del metabolismo fosfocálci$\mathrm{co}^{(15)}$.

Las variables paraclínicas analizadas en las dos poblaciones fueron concentraciones séricas de 25-hidroxi-vitamina D, calcio, fósforo, fosfatasa alcalina y PTHi.

La hipovitaminosis D se clasificó como insuficiencia de vitamina D si la concentración sanguínea era menor a $30 \mathrm{ng} / \mathrm{ml}$, déficit de vitamina $\mathrm{D}$ si era menor a 20 $\mathrm{ng} / \mathrm{ml}$, y déficit severo de vitamina $\mathrm{D}$ como la concentración sanguínea menor a $10 \mathrm{ng} / \mathrm{ml}^{(22)}$.

Los estudios paraclínicos se realizaron en un analizador Advia Centaur XP, Siemens Healthcare Diagnostics, por método de quimioluminiscencia, con controles BIORAD y calibradores Siemens.

Durante todo el estudio se mantuvo el anonimato de los datos personales de todos los participantes. El protocolo fue aceptado por el Comité de Ética del CASMU.

\section{Cálculo de la muestra}

Para el cálculo del tamaño de la muestra aleatoria el equipo de investigación consideró una proporción de hipovitaminosis D de $50 \%{ }^{(23)}$ y se obtuvo una muestra de 376 pacientes, teniendo presente además el nivel de confianza de $95 \%$ y un error alfa de $5 \%$. En niños se consideró también una proporción de hipovitaminosis D de $50 \%{ }^{(20)}$, siendo necesaria una muestra de 373 , con un nivel de confianza de $95 \%$ y un error alfa de $5 \%$. En caso de no llegar a este número, se realizará una muestra de conveniencia. 
Tabla 1. Características basales en niños $(n=60)$ y adultos $(n=53)$; CASMU 2017.

\begin{tabular}{|c|c|c|c|}
\hline Niños & Con $A E(n=22)$ & $\operatorname{Sin} A E(n=38)$ & Significancia estadística \\
\hline $\operatorname{Edad}(X \pm D S)$ & $8,63 \pm 3,86$ & $8,18 \pm 2,7$ & NS \\
\hline IMC (X; Mdn) & 17,$4 ; 17$ & 18,$9 ; 18$ & NS \\
\hline Sexo (F:M) & $06: 22$ & $20: 18$ & $p=0,03$ \\
\hline Adultos & Con AE $(n=21)$ & $\operatorname{Sin} A E(n=32)$ & Significancia estadística \\
\hline $\operatorname{Edad}(\mathrm{X} \pm \mathrm{DS})$ & $60,7 \pm 5,72$ & $59,46 \pm 5,49$ & NS \\
\hline IMC (X; Mdn) & $23 ; 23$ & 26,$8 ; 26$ & NS \\
\hline Sexo (F:M) & $10: 21$ & 18:14 & NS \\
\hline
\end{tabular}

Las variables de las características basales y de los AE se expresaron como frecuencias absolutas, relativas, media, mediana y rangos, según correspondiera. La prevalencia de hipovitaminosis se expresó como frecuencias absolutas, relativas y porcentajes. Los valores de hipovitaminosis se expresaron como media, desvío estándar (DE), mediana y rango. Para comparar los promedios de hipovitaminosis D se realizó comparación de medias por la prueba de $t$ para muestras independientes y las variables categóricas por chi cuadrado. La comparación de medianas se realizó mediante prueba de Mann- Whitney. Se consideró nivel de significancia estadística el valor de $\mathrm{p}<0,05$ para test de dos colas. Se utilizó el programa EpiInfo 3.5.

\section{Resultados}

Los datos se recabaron entre marzo y diciembre de 2017. No fue posible alcanzar el número de la muestra aleatoria calculada, porque no se halló la cantidad de personas necesarias en el tiempo estipulado para el estudio. Por lo tanto, se realizó una muestra por conveniencia que incluyó 113 pacientes: 60 niños (22 en tratamiento $\mathrm{AE}$ ) y 53 adultos ( 21 en tratamiento $\mathrm{AE}$ ). La media de edad de los niños fue de 9 años (mediana 8 años) y de los adultos 60 años (media y mediana). La media de IMC en niños fue de 18,4 (mediana 18) y de adultos 26,4 (mediana 26). No se presentaron diferencias en estas características entre los grupos expuestos y no expuestos a AE (tabla 1).

La prevalencia global de insuficiencia de vitamina D en el total de la población estudiada fue de $89 \%$ (101/113), y el déficit de vitamina D fue de $60 \%$ $(68 / 113)$.

Los AE utilizados por los pacientes incluidos fueron ácido valproico, carbamazepina y levetiracetam en el caso de los niños, y en caso de los adultos difenilhidantoína, carbamazepina, oxcarbazepina, ácido valproico y levetiracetam. En niños predominó el uso de ácido valproico y en adultos difenilhidantoína. Dos pacientes en el grupo de niños y dos en el de adultos tenían politerapia. La duración del tratamiento con $\mathrm{AE}$, tanto en niños como en adultos, fue más de seis meses en la mayoría de los casos (tabla 2).

La media de vitamina $D$ en niños expuestos a los $\mathrm{AE}$ fue $17,5 \mathrm{ng} / \mathrm{ml}$ (DE 8,7) y en niños no expuestos fue 19,6 $\mathrm{ng} / \mathrm{ml}$ (DE 6,8). La diferencia de medias no fue estadísticamente significativa (tabla 3$)$. El déficit $(<20 \mathrm{ng} / \mathrm{ml} \mathrm{y}$ $>10 \mathrm{ng} / \mathrm{ml}$ ) fue el grado de hipovitaminosis más comúnmente hallado, tanto entre los niños que recibían AE como en los que no, pero ningún grado de hipovitaminosis tuvo una diferencia estadísticamente significativa entre los grupos (tabla 4).

En adultos la media de vitamina $D$ fue de 18,1 en expuestos a $\mathrm{AE}(\mathrm{DE} 10,2)$ y 16,9 en no expuestos (DE 8,0). La diferencia de medias no fue significativa (tabla 3 ). Al igual que lo observado en niños, en adultos ningún grado de hipovitaminosis D fue estadísticamente significativo entre los grupos (tabla 3 ).

En pacientes que recibían $\mathrm{AE}$ se observaron niveles de calcemia descendidos de forma estadísticamente significativa menores que en quienes no los recibían, tanto en niños como en adultos (tabla 3 ), aunque en su mayoría dentro de rangos de normalidad. De los niños que recibían $\mathrm{AE}$, dos presentaron hipocalcemia (FR 0,09), uno presentaba insuficiencia y el otro déficit de vitamina $\mathrm{D}$. No se encontró hipocalcemia en niños no expuestos a AE. En adultos se observó hipocalcemia en dos pacientes expuestos (FR 0,95), uno de los cuales presentó déficit y otro insuficiencia de vitamina $\mathrm{D}$. En un paciente no expuesto a AE se observó hipocalcemia (FR 0,03 ) y presentaba además déficit de vitamina $\mathrm{D}$. Los niveles de 
Tabla 2. Tipo de antiepilépticos y duración del tratamiento en niños y adultos.

\begin{tabular}{lcccc}
\hline Antiepiléptico & Niños $\left(n=22^{*}\right)$ & Duración $(>6 \mathrm{~m})$ & Adultos $(n=19)^{*}$ & Duración $(>6 \mathrm{~m})$ \\
\hline Ácido valproico & 20 & 15 & 7 & 5 \\
Carbamazepina & 3 & 2 & 10 & 1 \\
Difenilhidantoína & 0 & 0 & 1 & 8 \\
Levetiracetam & 1 & 1 & 1 & 0 \\
Oxcarbazepina & 0 & 0 & & 0 \\
* Dos niños y dos adultos recibieron politerapia. & & &
\end{tabular}

Tabla 3. Niveles de vitamina D y metabolismo fosfo-cálcico en niños $(n=60)$ y adultos $(n=53)$, CASMU 2017.

\begin{tabular}{|c|c|c|c|c|c|}
\hline \multirow[t]{2}{*}{ Niños } & \multicolumn{2}{|c|}{ Con $A E(n=22)$} & \multicolumn{2}{|c|}{$\operatorname{Sin} A E(n=38)$} & \multirow[b]{2}{*}{ Valor $p$} \\
\hline & Media (DE) & Mediana (rango) & Media (DE) & Mediana (rango) & \\
\hline Vitamina D (ng/ml) & $17,59(8,70)$ & $15,7(4-34)$ & $19,67(6,81)$ & $20,10(9-36)$ & NS \\
\hline PTHi (pg/ml) & $42,64(18,23)$ & $38(19-84)$ & $39,92(20,1)$ & 36 (14-93) & NS \\
\hline Calcio (mg/dl) & $9,61(0,35)^{*}$ & $9,6(9-10)$ & $9,88(0,43)^{*}$ & $9,80(9-11)$ & $0,01521^{*}$ \\
\hline Fósforo (mg/dl) & $5,15(0,51)$ & $5,10(4-6)$ & $4,88(0,68)$ & $4,85(3-7)$ & NS \\
\hline \multirow[t]{2}{*}{ Adultos } & \multicolumn{2}{|c|}{ Con $A E(n=21)$} & \multicolumn{2}{|c|}{$\operatorname{Sin} A E(n=32)$} & \\
\hline & Media (DE) & Mediana (rango) & Media (DE) & Mediana (rango) & Valor $p$ \\
\hline Vitamina D (ng/ml) & $18,10(10,20)$ & $17,4(6-40)$ & $16,90(8,0)$ & $15(6-32)$ & NS \\
\hline PTHi (pg/ml) & $70,65(45,63)$ & $59(28-218)$ & $64,05(27,8)$ & $60(37-154)$ & NS \\
\hline Calcio (mg/dl) & $9,04(0,44)$ & $8,9(8-10)$ & $9,55(0,42)$ & $9,6(9-11)$ & $0.0000929^{*}$ \\
\hline Fósforo (mg/dl) & $3,60(0,66)$ & $3,6(2-5)$ & $3,33(0,64)$ & $3,40(2-4)$ & NS \\
\hline $\mathrm{FA}(\mathrm{mUl} / \mathrm{ml})$ & $91,76(30,01)$ & $86(45-154)$ & $90,68(32,42)$ & $87,5(47-178)$ & NS \\
\hline
\end{tabular}

* p significativa para la comparación de medias. AE: antiepilépticos; DE: desvío estándar; PTHi: parathormona intacta; FA: fosfatasa alcalina.

PTH, fósforo y fosfatasa alcalina no presentaron diferencias estadísticamente significativas entre grupos (tabla 3).

\section{Discusión}

El presente es el primer estudio uruguayo en describir la prevalencia de hipovitaminosis D en una población que incluye niños y adultos. Se encontró una prevalencia global elevada de insuficiencia de vitamina $\mathrm{D}$, cercana a $90 \%$, y un déficit global de $60 \%$, con medias de vitamina $\mathrm{D}$ en todos los grupos menores o iguales a $20 \mathrm{ng} / \mathrm{ml}$. Estos datos concuerdan con estudios nacionales e internacionales ${ }^{(3-11)}$. Un estudio realizado en Uruguay en
2015 en adultos sanos $(n=175)$ que concurrían a realizarse el carné de salud, encontró niveles de insuficiencia de vitamina $\mathrm{D}$ en el $89 \%$ de la población y la media de vitamina $\mathrm{D}$ encontrada fue de $17,8 \mathrm{ng} / \mathrm{ml}^{(7)}$. Otro estudio nacional que incluyó mujeres embarazadas cursando el tercer trimestre mayores de 18 años que fueron internadas en el Centro Hospitalario Pereira Rossell $(\mathrm{n}=71)$, durante abril a setiembre de 2014, encontró una prevalencia de insuficiencia de vitamina D de $96 \%$, con $42 \%$ de déficit y $27 \%$ de déficit severo ${ }^{(9)}$. Otro estudio incluyó 105 pacientes en hemodiálisis crónica y encontró valores de vitamina $\mathrm{D}$ menores a $30 \mathrm{ng} / \mathrm{ml}$ en $97 \%$ de la población, con una media de vitamina $\mathrm{D}$ de 13 
Tabla 4. Prevalencia de hipovitaminosis en niños y adultos, CASMU $2017(n=113)$.

\begin{tabular}{lcccc}
\hline & $\begin{array}{c}\text { Insuficiencia } \\
(<30 \mathrm{ng} / \mathrm{ml}>20 \mathrm{ng} / \mathrm{ml})\end{array}$ & $\begin{array}{c}\text { Déficit } \\
(<20 \mathrm{ng} / \mathrm{ml}>10 \mathrm{ng} / \mathrm{ml})\end{array}$ & $\begin{array}{c}\text { Déficit severo } \\
(<10 \mathrm{ng} / \mathrm{ml})\end{array}$ & $\begin{array}{c}\text { Hipovitaminosis global } \\
(<30 \mathrm{ng} / \mathrm{ml})\end{array}$ \\
\hline Niños con AE (N=22) & 4 & 12 & 3 & $19(86 \%)$ \\
Niños sin AE (N=38) & 16 & 17 & 2 & $35(92 \%)$ \\
Adultos con AE (N=21) & 6 & 5 & 7 & $18(86 \%)$ \\
Adultos sin AE (N=32) & 7 & 16 & 18 & $29(90 \%)$ \\
Total & 33 & 50 & & $101 / 113$ \\
\hline AE: antiepilépticos. & & & & \\
\hline
\end{tabular}

$\mathrm{ng} / \mathrm{ml}^{(8)}$. Como se puede observar, los datos nacionales refieren a adultos o a poblaciones especiales, que pueden determinar a priori un riesgo mayor de hipovitaminosis D.

La significancia clínica de los valores hallados tanto en este estudio como en los citados como referencias bibliográficas, es una cuestión en debate, más aun considerando la variabilidad en el establecimiento de los puntos de corte para determinarlos ${ }^{(11)}$. Es importante continuar analizando las implicancias de estos hallazgos y la necesidad de suplementación preventiva en función del balance beneficio/riesgo que esto implica.

En este estudio el período de toma de muestras fue extenso, abarcando la mayoría de las estaciones del año, dejando de lado así las conocidas influencias estacionales sobre los valores sanguíneos de vitamina $\mathrm{D}$, a diferencia de otros estudios nacionales que incluyeron exclusivamente períodos invernales ${ }^{(7,8)}$.

En cuanto a las características basales no se encontraron diferencias entre grupos según edad, sexo e IMC, (esta consideración es sobre las muestras de estudio; habla de la validez intrínseca y del diseño de este estudio, no corresponde su comparación con la literatura) $)^{(7-11)}$. El rango etario elegido de adultos presenta una proporción de pacientes que incluye toda la población considerada adulto mayor ( $>65$ años), lo cual puede influir en los bajos niveles de vitamina D hallados ${ }^{(24,25)}$. Este rango etario fue seleccionado para disminuir las diferencias que existen entre hombres y mujeres debido a las influencias hormonales en las mujeres premenopáusicas. En el caso de los niños, se eligieron mayores de 2 años, dado que en menores de esta edad se realiza suplementación con vitamina D reglada según pautas nacionales, debido al riesgo de raquitismo que existe en esta edad ${ }^{(26,27)}$. En el grupo de niños que recibían $\mathrm{AE}$ se encontró una mayor proporción de varones, lo cual es acorde al discreto predominio de epilepsia en el sexo masculino en la edad pediátrica $^{(28)}$.
No se identificó una diferencia estadísticamente significativa entre el grupo que recibía $\mathrm{AE}$ y el que no, en ninguno de los rangos etarios evaluados. Esto puede deberse a que el número de pacientes incluidos en el estudio no alcanzó el tamaño muestral calculado previamente y a la elevada prevalencia encontrada de déficit e insuficiencia de vitamina $D$, pudiendo así subestimar el efecto de los AE sobre ésta. Como ha sido valorado en varios estudios existen otras variables que podrían estar influyendo en los valores de vitamina $\mathrm{D}$, como la exposición solar con o sin pantallas, la ingesta de vitamina $\mathrm{D}$ en la dieta, la edad, el antecedente de falta de suplementación en el caso de niños ${ }^{(29-33)}$. Otros autores, como Borusiak y colaboradores, encontraron similares hallazgos en un estudio prospectivo de niños menores de 18 años (media de edad 9 años) con diagnóstico de epilepsia, también con una muestra pequeña $(\mathrm{n}=128,76$ niñas y 52 varones). Los AE utilizados en ese estudio fueron VPA, CBZ, oxcarbazepina, topiramato, lamotrigina y levetiracetam y la duración media del tratamiento de 30 meses (6 meses a 3 años). Las diferencias en vitamina $\mathrm{D}$ no fueron significativas con el grupo control, presentando todos los pacientes niveles inferiores a $30 \mathrm{ng} / \mathrm{ml}^{(15)}$. Nicolaidou y colaboradores en un estudio observacional, prospectivo, controlado, realizado en niños epilépticos $(n=51)$ en tratamiento con CBZ y VPA, encontraron diferencias significativas en los niveles de vitamina D cuando se consideraron las estaciones del año, observándose niveles menores en invierno versus verano y otoño versus primavera, y con un tiempo de tratamiento con AE mayor a un año. No hallaron diferencias significativas en pacientes tratados por menos de un año. Se observó $49 \%$ de hipovitaminosis menor a $10 \mathrm{ng} / \mathrm{dl}$ en la población estudiada, encontrando una tendencia decreciente de vitamina $\mathrm{D}$ a medida que aumentaba el tiempo de uso de $\mathrm{AE}^{(31)}$. Este hallazgo es interesante pues implica un posible efecto a largo plazo que se debería continuar analizando. 
En el presente estudio la duración del tratamiento antiepiléptico fue en su mayoría mayor a 6 meses, siendo similar a los hallazgos de otros autores ${ }^{(15,31,32)}$.

Por otro lado, al analizar otro de los componentes relevantes del metabolismo fosfocálcico como es la calcemia, se encontraron diferencias significativas, ya que los pacientes expuestos a AE presentaron niveles de calcemia inferiores a los pacientes no expuestos. Este hecho se constató tanto en la población pediátrica como en adultos (tabla 3). Este descenso determinó hipocalcemia solo en algunos pacientes, según los parámetros de laboratorio utilizados. Borusiak y colaboradores en su estudio encontraron hallazgos similares, siendo la hipocalcemia en pacientes expuestos a $\mathrm{AE}$ significativamente más frecuente que en los controles. Definió hipocalcemia como concentraciones por debajo del tercer percentil de valores de referencia específicos de la edad y la observó en $24,4 \%$ de los pacientes que recibían $\mathrm{AE}$ (media $9,65 \mathrm{mg} / \mathrm{dl}$ ) en relación con los controles sanos (media $10,1 \mathrm{mg} / \mathrm{dl})^{(15)}$. Estos hallazgos podrían explicarse como alteraciones iniciales del metabolismo fosfocálcico, posiblemente por una acción directa de los AE sobre la absorción intestinal de calcio, la cual se ha observado sobre todo con AE inductores, y en especial con fenitoína. A su vez, aquellos pacientes cuyos niveles descendidos de calcio llegaron a niveles de hipocalcemia, todos tenían hipovitaminosis, sumándose en estos pacientes el efecto de la disminución de vitamina $\mathrm{D}$ biológicamente activa sobre el intestino, alterando así la absorción de calcio $^{(34,35)}$. Algo similar fue observado en este trabajo.

En vistas a continuar analizando los efectos de los AE sobre el metabolismo fosfocálcico y la vitamina D sería importante hacer estudios con una muestra mayor, así como abarcar más rangos etarios y en otras poblaciones de Uruguay. Sería interesante conocer la influencia de la estacionalidad y continuar caracterizando otras variables como el IMC, la raza, las características de exposición solar, la dieta, etc. Otro aspecto a evaluar es el efecto de la suplementación con vitamina D y calcio en pacientes con déficit, porque esto redundaría en recomendaciones con mejor nivel de evidencia que las actualmente disponibles ${ }^{(22,36)}$. También sería relevante conocer el impacto clínico de los hallazgos encontrados y correlacionarlo con alteraciones de la densidad mineral ósea, su influencia sobre riesgo de fracturas y eventuales consecuencias extraóseas.

\section{Conclusiones}

El déficit de vitamina $\mathrm{D}$ hallado en esta población globalmente supera el 50\%, tanto en niños como en adultos, y es independiente del uso de AE. Sin embargo, esta falta de relación con el uso de AE no puede asegurarse por los datos de este estudio, dado que la muestra anali- zada de pacientes fue insuficiente. El único hallazgo con significación estadística fue el de los valores de calcio descendidos en los pacientes que utilizaban AE. Es necesario continuar analizando los múltiples factores que pueden determinar estos valores de vitamina $\mathrm{D}$ en la población uruguaya, su vinculación con la hipocalcemia y las consecuencias clínicas de estos hallazgos. Este tipo de evidencia contribuiría a la toma de decisiones sobre las recomendaciones de prevención y tratamiento con vitamina D.

\section{Agradecimientos}

A los Dres. Stéfano Fabbiani, Federico Garaffoni, Dévora Pérezy Gustavo Arroyo.

\section{Summary}

Introduction: hypovitaminosis D is a highly spread condition worldwide, with clinical consequences that affect bone directly, among other manifestations. Antiepileptic drugs are among factors that cause this deficiency. In Uruguay, there is no information about hypovitaminosis $\mathrm{D}$ in children or patients who receive antiepileptic drugs.

Objectives: to learn about the prevalence of hypovitaminosis D in children and adults in a health institution and to compare it with the prevalence in patients receiving antiepileptic drugs.

Method: descriptive, transversal study conducted from March through December, 2017. The following variables were analysed: vitamin $\mathrm{D}$, calcium, phosphorous, alkaline phosphatase and intact parathyroid hormone. Vitamin D insufficiency was defined as vitamin D levels of less than $30 \mathrm{ng}$ per $\mathrm{mL}$ and deficiency as D levels of less than $20 \mathrm{ng}$ per $\mathrm{mL}$.

Results: 113 patients were included in the study, 60 of which were children and 53 adults. Global prevalence of vitamin D insufficiency was $89 \%$ and deficiency was $60 \%$. In children taking antiepileptic drugs, the average vitamin D value was $17.5 \mathrm{ng} / \mathrm{ml}$ and it was $19.6 \mathrm{ng} / \mathrm{ml}$ for those not exposed to those drugs. In adults, the average vitamin D value was 18.1 in the population taking antiepileptic drugs and 16.9 in patients not taking that medication. The difference between average values was not statistically significant in children or adults. Calcemia levels observed were significantly lower in both children and adults taking antiepileptic drugs.

Conclusions: vitamin D insufficiency was close to $90 \%$ and deficiency was over $50 \%$. No significant differences were found between hypovitaminosis D groups, although reduced calcemia was observed in patients exposed to antiepileptic drugs. Further studies are necessary to analyse factors that cause this condition and its clinical consequences. 


\section{Resumo}

Introdução: a hipovitaminose D está amplamente difundida em todo o mundo, com consequências clínicas a nível ósseo e extraósseo. Entre os fatores que a causam estão os medicamentos antiepilépticos (AE). No Uruguai, sua prevalência em crianças ou em pacientes adultos recebendo $\mathrm{AE}$ não é conhecida.

Objetivos: conhecer a prevalência de hipovitaminose D em crianças e adultos em um prestador de serviços de saúde e compará-la com a prevalência em pacientes em tratamento com $\mathrm{AE}$.

Método: estudo transversal descritivo realizado entre março e dezembro de 2017. As variáveis analisadas foram: níveis de vitamina $\mathrm{D}$, cálcio, fósforo, fosfatase alcalina e paratormona intacta. Níveis menores que 30 $\mathrm{ng} / \mathrm{ml}$ e níveis de déficit menores que $20 \mathrm{ng} / \mathrm{ml}$ foram considerados como insuficiência de vitamina D.

Resultados: foram incluídos 113 pacientes, 60 crianças e 53 adultos. A prevalência global de insuficiência de vitamina D foi de $89 \%$ e déficit de $60 \%$. Em crianças expostas à $\mathrm{AE}$, a média de vitamina $\mathrm{D}$ foi de $17,5 \mathrm{ng} / \mathrm{ml}$ e em crianças não expostas de 19,6 ng / ml. Em adultos, a média de vitamina $\mathrm{D}$ foi de 18,1 nos expostos ao $\mathrm{AE}$ e de 16,9 nos não expostos. A diferença nas médias não foi estatisticamente significativa nas crianças nem nos adultos. Níveis de cálcio significativamente diminuídos foram observados em crianças e adultos com EA.

Conclusões: a insuficiência de vitamina $\mathrm{D}$ foi próxima a $90 \%$ e o déficit ultrapassou $50 \%$. Não foram encontradas diferenças significativas entre os grupos na hipovitaminose $\mathrm{D}$, mas níveis reduzidos de cálcio foram observados naqueles expostos a EA. É necessário continuar analisando os fatores que o causam e suas consequências clínicas.

\section{Bibliografía}

1. Vitamina D: evidencias y controversias. Bol INFAC 2012; 20(2):1-6. Disponible en: https://www.euskadi.eus/contenidos/informacion/cevime_infac_2012/es_def/adjuntos/INFAC_Vol_20_n_2.pdf [Consulta: 12 enero 2018].

2. Mithal D, Wahl DA, Bonjour JP, Burckhardt P, Dawson-Hughes B, Eisman JA, et al. Global vitamin D status and determinants of hypovitaminosis D. Osteoporos Int 2009; 20:1807-20.

3. Ganji V, Zhang X, Tangpricha V. Serum 25-hydroxivitamin D concentrations and prevalence estimates of hypovitaminosis D in the U.S. population based on assay-adjusted data. J Nutr 2012; 142(3):498-507.

4. Aparicio A, López-Sobaler A, López Plaza B, Perea J, Ortega R. Ingesta de vitamina $D$ en una muestra representativa de la población española de 7 a 16 años: diferencias en el aporte y las fuentes alimentarias de la vitamina en función de la edad. Nutr Hosp 2013; 28(5):1657-65.

5. Ruiz-Ocaña $\mathbf{P}$, Carrasco-Fernández $\mathbf{C}$, Zopeque-García N, Saez-Benito A, Novalbos-Ruiz J, Lechuga-Sancho A. Niveles de vitamina $\mathrm{D}$ al final de la estación invernal en una población escolar sana. Rev Esp Endocrinol Pediatr 2014; 5:19-27.

6. Puche R. Sobre la prevalencia de hipovitaminosis D en Argentina. Medicina (Buenos Aires) 2015;75(3):183-6.

7. Bagattini J, Barrios E, Barañano R, Moratorio G, Montes J, Laporte S, et al. Estado de la vitamina D en adultos uruguayos aparentemente saludables, en invierno y en Montevideo. Rev Méd Urug 2017; 33(2):126-37.

8. Solá L, Leyún N, Díaz JC, González S, Sehabiague C, Parodi $\mathrm{K}$, et al. Asociación de déficit de vitamina $\mathrm{D}$ y anemia en pacientes en hemodiálisis crónica. Rev Méd Urug 2014; 30(3):157-63.

9. Gómez F, Bottaro S, Cóppola F, Tomasso G, Rocha V, Giménez $C$. Prevalencia del déficit de vitamina $D$ en una población de embarazadas que se asisten en el Centro Hospitalario Pereira Rossell. Rev Méd Urug 2016; 32(2):87-97.

10. Mendoza B, Ronco A. Estacionalidad de la vitamina D plasmática: su importancia en la clínica y en la salud. Rev Méd Urug 2016; 32(2):80-6.

11. De Santis A, Catenaccio V, Speranza N. Hipovitaminosis D y antiepilépticos: revisión de la evidencia y recomendaciones. Rev Méd Urug 2019; 35(3):224-31.

12. Beerhost K, Van der Kruijs S, Verschuure P, Tan IY, Aldenkamp A. Bone disease during chronic antiepileptic drug therapy: general versus specific risk factors. J Neurol Sci 2013; 331:19-25.

13. Tomita S, Ohnishi J, Nakano M, Ichikawa Y. The effect of anticonvulsant drugs on vitamin D3-activating cytochrome P-450-linked monooxygenase systems. J Steroid Biochem Mol 1991; 39:479-85.

14. Farhat G, Yamout B, Mikati MA, Demirjian S, Sawaya R, El-Hajj Fuleihan G. Effect of antiepileptic drugs on bone density in ambulatory patients. Neurology 2002; 58:1348-53.

15. Borusiak P, Langer T, Heruth M, Karenfort M, Bettendorf U, Jenke A. Antiepileptic drugs and bone metabolism in children: data from 128 patients. J Child Neurol 2012; 28(2):176-83.

16. Gniatkowska-Nowakowska A. Fractures in epilepsy children. Seizure 2010; 19(6):324-5.

17. Teagarden D, Meador K, Loring D. Low vitamin D levels are common in patients with epilepsy. Epilepsy Res 2014; 108(8): 1352-6.

18. Miratashi S, Abbasi M, Miratashi S. Epilepsy and vitamin D: a comprehensive review of current knowledge. Rev Neurosci 2017; 28(2):185-201.

19. Organización Mundial de la Salud. 10 datos sobre la obesidad. Disponible en: https://www.who.int/features/factfiles/obesity/facts/es/ [Consulta: 2 noviembre 2020].

20. WHO Collaborating Centre for Drugs Statistics Methodology. ATC/DDD Index 2021. Disponible en: 
http://www.whocc.no/atc_ddd_index/[Consulta: 10 mayo 2020].

21. García-Peñas J, Jiménez-Legido M. Politerapia racional en epilepsia infantil. Pediátr Panamá 2017; 46(2):82-6.

22. Holick M, Binkley N, Bischoff-Ferrari H, Gordon C, Hanley D, Heaney R, et al. Evaluation, treatment, and prevention of vitamin d deficiency: an endocrine society clinical practice guideline. J Clin Endocrinol Metab 2011; 96(7):1911-30.

23. Sánchez A, Oliveri B, Mansur J, Fradinger E. Diagnóstico, prevención y tratamiento de la hipovitaminosis D. RAEM 2013; 50(2):142-57.

24. Uruguay. Ministerio de Salud Pública, Dirección General de la Salud. Programa Nacional del Adulto Mayor. Montevideo: MSP, 2005: 36p. Disponible en: https://www.gub. uy/ministerio-salud-publica/sites/ministerio-salud-publica/files/documentos/publicaciones/Programa\%20Adulto\%20Mayor.pdf. [Consulta: 16 febrero 2020].

25. Serrano J, Latorre J, Gatz M. Spain: promoting the welfare of older adults in the context of population aging. Gerontologist 2014; 54(5):733-40.

26. Uruguay. Ministerio de Salud Pública. Suplementación con vitamina D en niños. Montevideo: MSP, 2017. Disponible en: https://www.gub.uy/ministerio-salud-publica/comunicacion/comunicados/suplementacion-con-vitamina-d-en-ninos [Consulta: 22 febrero 2020].

27. Atención pediátrica: normas nacionales de diagnóstico, tratamiento y prevención. 8 ed. Montevideo: Oficina del Libro FEFMUR, 2014
28. Aaberg K, Gunnes N, Inger J. Incidence and prevalence of childhood epilepsy: a nationwide cohort study. Pediatrics 2017; 139(5):1-9.

29. De Souza Silva J, Pereira SE, Saboya Sobrinho CJ, Ramalho A. Obesity, related diseases and their relationship with vitamin D deficiency in adolescents. Nutr Hosp 2016; 33:381.

30. Santos Araújo EPD, Queiroz DJM, Neves JPR, Lacerda LM, Gonçalves MDC, Carvalho AT. Prevalence of hypovitaminosis D and associated factors in adolescent students of a capital of northeastern Brazil. Nutr Hosp 2017; 34:1416-23.

31. Nicolaidou P, Georgouli H, Kotsalis H, Matsinos Y, Papadopoulou A, Fretzayas A. Effects of anticonvulsant therapy on vitamin $\mathrm{D}$ status in children: prospective monitoring study. J Child Neurol 2006; 21(3):205-9.

32. Fong CY, Kong AN, Poh BK, Mohamed AR, Khoo TB, Ng RL, et al. Vitamin D deficiency and its risk factors in Malaysian children with epilepsy. Epilepsia 2016; 57(8):1271-9.

33. Baek J, Seo Y, Kim G, Kim M, Eun B. Vitamin D levels in children and adolescents with antiepileptic drug treatment. Yonsei Med J 2014; 55(2):417-21.

34. Pack A, Gidal B, Vazquez B. Bone disease associated with antiepileptic drugs. Cleve Clin J Med 2004; 71(Suppl 2):S42-8.

35. Hamed S. Markers of bone turnover in patients with epilepsy and their relationship to management of bone diseases induced by antiepileptic drugs. Expert Rev Clin Pharmacol 2016; 9:267-86.

36. Golden NH, Abrams SA; Committee on Nutrition. Optimizing bone health in children and adolescents. Pediatrics 2014; 134(4):e1229-43.

\footnotetext{
Contribución de autores

Todos los autores participaron en igual medida en las distintas etapas de elaboración del artículo. Valentina Catenaccio, ORCID 0000-0003-4236-610X

Silvia Xavier, ORCID 0000-003-2805-1793

Agustina De Santis, ORCID 0000-0002-6630-0925

Florencia Galarraga , ORCID 0000-0001-5775-4231

Noelia Speranza, ORCID 0000-0002-3330-9974

Andrés Bálsamo, ORCID 0000-0002-8289-1921

Luciana Salazar, ORCID 0000-0002-6273-9420

Eugenia Chaibun, ORCID 0000-0003-1631-236X

Ricardo Hermo, ORCID 0000-0001-8444-663X

Laura Solá, ORCID 0000-0003-3269-3908

Stella Gutiérrez, ORCID 0000-0001-7529-788X

Gustavo Tamosiunas, ORCID 0000-0002-3619-9651
} 